Gut, 1988, 29, 70-74

\title{
Which peptic ulcer patients bleed?
}

\author{
K MATTHEWSON, S PUGH, AND T C NORTHFIELD \\ From the Gastroenterology Units, St James Hospital, Balham and University College Hospital, London
}

SUMMARY In order to identify factors associated with peptic ulcers which present with bleeding rather than with pain, we have prospectively evaluated patient and endoscopic features in 139 consecutive patients presenting with acute bleeding and 74 presenting with pain found to have peptic ulceration at endoscopy. Patients with bleeding were more likely to have taken nonsteroidal anti-inflammatory drugs within the preceding four weeks $(58 \% v 18 \%, \mathrm{p}<0 \cdot 001)$. They were older $(66 v 51$ years, $\mathrm{p}<0 \cdot 001)$, more likely to have had a previous ulcer complication $(21 \% v$ $1 \%, \mathrm{p}<0.001)$, and to have an ulcer diameter greater than $20 \mathrm{~mm}(27 \% v 6 \%, \mathrm{p}<0.05)$. The presence of one or more of these features in a patient who develops a peptic ulcer appears to be associated with a greater likelihood of presentation with a gastrointestinal haemorrhage.

Peptic ulceration can present in a number of ways, the two commonest being pain and acute bleeding. Despite the fact that acute gastrointestinal bleeding is a potentially fatal complication, there is little information regarding factors which influence a particular patient's ulcer to present with bleeding rather than with pain. Attention has recently been drawn to the association between the use of non-steroidal antiinflammatory drugs (NSAIDs) and bleeding peptic ulcer,' but it is not clear whether their use is associated specifically with peptic ulcers that present with bleeding rather than with pain. Smoking is also associated with peptic ulceration in outpatients, ${ }^{2}$ but whether the association is stronger with non-bleeding or bleeding peptic ulcers is likewise not known.

Peptic ulcers may be single or multiple; they occur in a variety of sites, their sizes vary considerably and they may be acute and superficial with soft bases or chronic and deep with hard fibrous bases. Whether these variations in ulcer appearance influence the way in which the ulcer presents is not known.

In a prospective study we have therefore compared patient features (age, sex, smoking, alcohol consumption, NSAID use and previous history of peptic ulceration) and endoscopic features (ulcer number, site, diameter, depth, nature of ulcer base and presence or absence of stigmata of recent haemorr-

Address for correspondence: Dr K Matthewson, Senior Registrar in Medicine. Southampton General Hospital. Tremona Rd. Southampton SO9 4XY.

Received for publication 2 July 1987. hage) in a consecutive series of patients presenting principally with acute bleeding and in another presenting with pain, all being found to have peptic ulcers at endoscopy.

\section{Methods}

PATIENTS

Between 1 September, 1984 and 28 October, 1986, one of us (KM), was continually on call to do emergency endoscopies at St James Hospital, Balham and University College Hospital, London. Of a consecutive series of patients admitted with acute upper gastrointestinal haemorrhage and referred to him for diagnostic endoscopy, 139 were thought to have bled from a peptic ulcer, which for the purposes of this study was defined as a break in the mucosa greater than $5 \mathrm{~mm}$ in diameter. After careful endoscopic washing of the ulcer base to remove loosely adherent overlying debris, the following information was collected from these 139 patients: number of ulcers, site, diameter, depth, nature of ulcer base, and presence or absence of stigmata of recent haemorrhage (SRH).

Ulcer site was categorised as one of the following: fundus, lesser curve, greater curve, antrum, stoma, duodenal bulb or postbulbar. Ulcer diameter was measured by opening a pair of biopsy forceps of known span in front of the ulcer. Both the largest and smallest diameters of the ulcer were recorded and the mean of these was taken as the ulcer diameter. If 
there were multiple ulcers, then the diameter of all ulcers were recorded. In the absence of active bleeding, a judgment was made as to which ulcer was the source of bleeding and this was regarded as the principal ulcer. This judgment was made on the basis of the presence of SRH in the base of each ulcer - that is, the presence of a visible vessel, overlying clot or red or black spots. In the presence of multiple ulcers without SRH, the largest ulcer was regarded as the principal ulcer.

Ulcer depth was determined subjectively and expressed numerically using the following scale: $0=$ ulcer level with surrounding mucosa; $1=$ ulcer crater just visible; $2=$ moderately deep crater; $3=$ very deep ulcer crater. The ulcer base was described as fibrous if it appeared yellow, hard and craggy, and non-fibrous if it appeared white and soft. These impressions were not confirmed histologically because it is not our practice to biopsy patients with acutely bleeding peptic ulcers.

When the patients had recovered sufficiently from the sedation given for endoscopy, they were interviewed and the following information was recorded: age, sex, cigarette smoking habits, ethanol consumption, NSAID use and the presence of a previous ulcer diagnosis.

Patients were regarded as NSAID users if they had consumed any of these drugs within the preceding four weeks, including the use of non-aspirin NSAIDs (NANSAIDs) and the use of aspirin. The types of

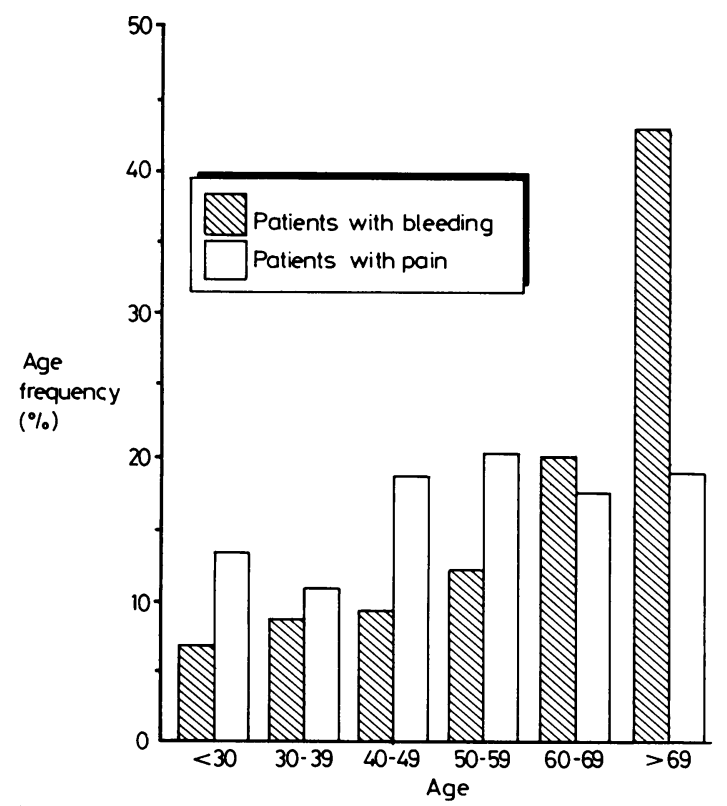

Fig. 1 Age distribution in patients presenting with bleeding and pain.
NANSAID used were noted. Previous ulcer diagnoses were recorded only if they had been confirmed radiologically or endoscopically. In patients with a previous ulcer diagnosis, the mode of its presentation was recorded. Details of NSAID use and previous ulcer diagnosis were corroborated where possible from the patient's notes and the referring doctor's letter.

During the same period of time, all 74 patients presenting with pain, who were endoscoped on the routine endoscopy lists of KM and SP at the same two hospitals and found to have peptic ulceration, were studied. Patients presenting several times during this period were studied only on the first presentation. The endoscopic and patient details recorded for these patients were identical to those for the patients with acute bleeding, with the exception that in the presence of multiple ulceration the largest ulcer was always regarded as the principal one. To ensure uniformity of interpretation of endoscopic findings, $\mathrm{SP}$ was present during endoscopy of some of the early patients with bleeding.

Comparison of numeric variables was performed using the Wilcoxon's rank-sum test and comparison of proportions using the $\chi^{2}$ test with Yates continuity correction.

\section{Results}

Patients presenting with bleeding were significantly older than patients with pain (median $66 v 51$ years, $\mathrm{p}<0.001$ ), and the breakdown of age distribution in the two groups is shown in Figure 1. Because of this age difference other statistical comparisons were age corrected. Cochrane's method was used with age subdivisions $0-39,40-59$, and $60+$. These subdivisions were not predefined but were not based on examination of the data set. Patients with bleeding were more likely to have used NSAIDs within the previous four weeks $\left(58 \% \vee 18 \%, \chi^{2}=21 \cdot 3\right.$, $\mathrm{p}<0 \cdot 001)$. The breakdown of NANSAID and aspirin use by age and presentation is shown in Figure 2. The differences in the use of NSAIDs between patients with bleeding and those with pain were significant even when the use of NANSAIDs $(34 \% v 5 \%$, $\left.\chi^{2}=9.1, \mathrm{p}<0.01\right)$ and the use of aspirin $(24 \% v 13 \%$, $\left.\chi^{2}=12 \cdot 6, \quad p<0 \cdot 001\right)$ were considered separately. The difference in age between the two groups was apparent even when all NSAID users were excluded from the analysis (median $65 v 50, \mathrm{p}<0 \cdot 001$ ) and was therefore not a consequence of the tendency for NANSAIDs to be given mainly to the elderly. The varieties of NANSAIDs used are shown in the Table.

There was a significantly lower frequency of smoking in patients with bleeding rather than with pain $\left(33 \% \vee 56 \%, \chi^{2}=4 \cdot 29, \mathrm{p}<0 \cdot 05\right)$ but no 

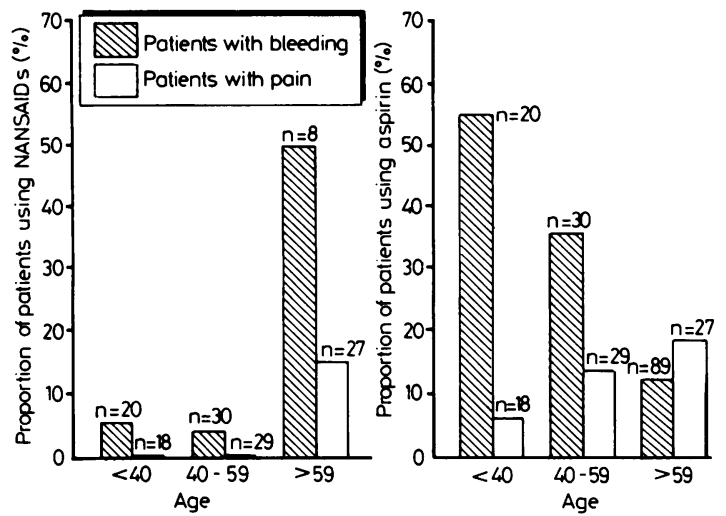

Fig. 2 Percentages of patients with bleeding or pain using NANSAIDs or aspirin by age.

significant differences in the male:female ratio $(1 \cdot 7: 1$ $v 2: 1, \chi^{2}=0 \cdot 69$, NS), the rate of ethanol consumption (median $0 \vee 5 \mathrm{~g} /$ week, NS) or the frequency of a previous ulcer diagnosis ( $32 \% v 48 \%, \chi^{2}=3 \cdot 4$, NS). In those patients with a previous ulcer diagnosis there were marked differences in the modes of the previous presentation between those currently presenting with bleeding or pain. Thus in those currently presenting with bleeding there was a significantly greater preceding frequency of bleeding $\left(46 \% v 2 \%, \chi^{2}=11.5\right.$, $\mathrm{p}<0.001$ ), a strong trend towards an increased preceding frequency of perforation $\left(20 \% v 0 \%, \chi^{2}=2 \cdot 5\right.$, NS) and a significantly lower preceding frequency of pain $\left(33 \% v 94 \%, \chi^{2}=21.2, \mathrm{p}<0.001\right)$. Considering the incidence of a previous ulcer complication (bleeding or perforation) in all patients irrespective of whether there was a previous ulcer diagnosis, there was a significantly greater frequency in those currently with bleeding than with pain $(21 \% v 1 \%$, $\left.\chi^{2}=11 \cdot 2, \mathrm{p}<0 \cdot 001\right)$.

Fifty one per cent of patients who had presented with bleeding also admitted to pain when specifically questioned. The absence of pain was not related to NSAID use ( $49 \%$ of NSAID users $v 48 \%$ of NSAID non-users, $\left.\chi^{2}=0 \cdot 74, \mathrm{NS}\right)$, but was related to age $(62 \%$

Table Numbers of patients with bleeding and pain using particular types of NANSAID

\begin{tabular}{lcl}
\hline NANSAID (type) & $\begin{array}{l}\text { Patients with } \\
\text { bleeding }(n)(\%)\end{array}$ & $\begin{array}{l}\text { Patients with } \\
\text { pain }(n)\end{array}$ \\
\hline Flurbiprofen & $4(9 \%)$ & 0 \\
Ibuprofen & $5(11 \%)$ & 1 \\
Indomethacin & $6(13 \%)$ & 0 \\
Naproxen & $12(26 \%)$ & 1 \\
Piroxicam & $9(20 \%)$ & 2 \\
Others & $10(22 \%)$ & 0 \\
Total & $46(100 \%)$ & 4 \\
\hline
\end{tabular}

of those over 70 years $v 37 \%$ of those under 70 years, $\left.\chi^{2}=7.41, p<0.01\right)$. Forty per cent of the patients with bleeding but no pain who had a previous ulcer diagnosis had previously presented with pain but no bleeding.

The distribution of ulcer diameters in each group is shown in Figure 3. The median diameter of the principal ulcer in patients with bleeding was significantly greater than in those with pain $(10 v 8.5 \mathrm{~mm}$, $\mathrm{p}<0 \cdot 01)$. There were significantly more patients with bleeding and large ulcers (diameter $20 \mathrm{~mm}$ or greater) than patients with pain and large ulcers $\left(27 \% \vee 6 \%, \chi^{2}=5.5, \mathrm{p}<0.05\right)$. In bleeding patients there was no significant difference in the proportion of large ulcers in NSAID users and non-users (32\% $v$ $19 \%, \chi^{2}=1 \cdot 2$, NS). The latter comparison could not be carried out in patients presenting with pain as there were few large ulcers in this group.

There was no difference in the incidence of multiple ulceration between ulcer patients presenting with bleeding or pain $\left(28 \% v 20 \%, \chi^{2}=2 \cdot 7, \mathrm{NS}\right)$; nor were there significant differences in ulcer depth (median depth $2 v 2$, NS), in the incidence of fibrous based ulcers $\left(56 \% \vee 61 \%, \chi^{2} 1.9\right.$, NS), or in the frequency of the various ulcer sites which are shown in Figure 4 . There was a significantly greater frequency of antral ulceration in NSAID users than nonusers when bleeding patients were considered alone $\left(15 \% v 1 \%, \chi^{2}=6 \cdot 8, \mathrm{p}<0.01\right)$ and when all patients

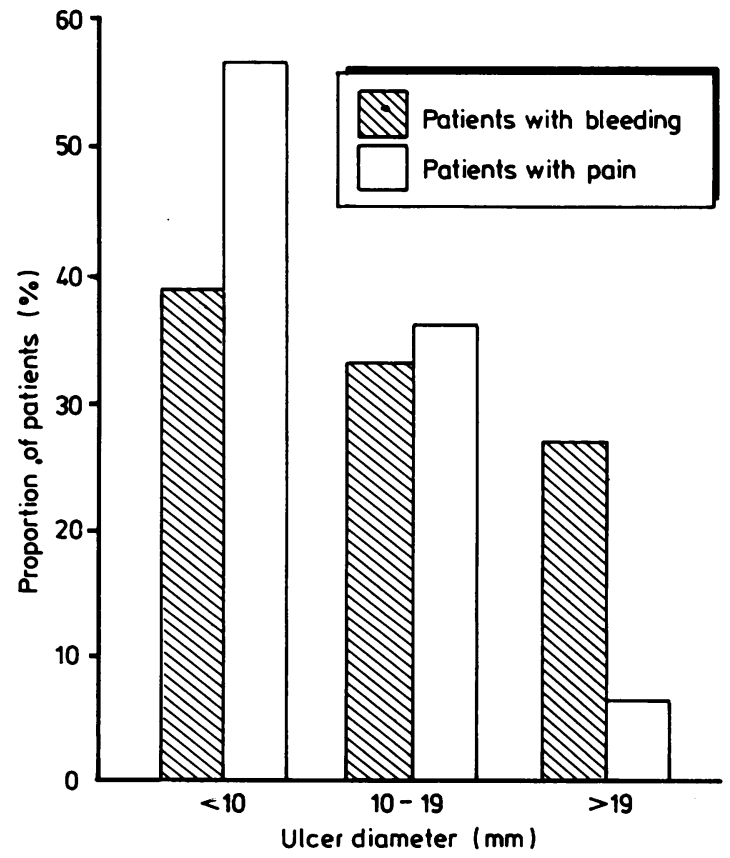

Fig. 3 Distribution of ulcer diameters in patients with bleeding or pain. 


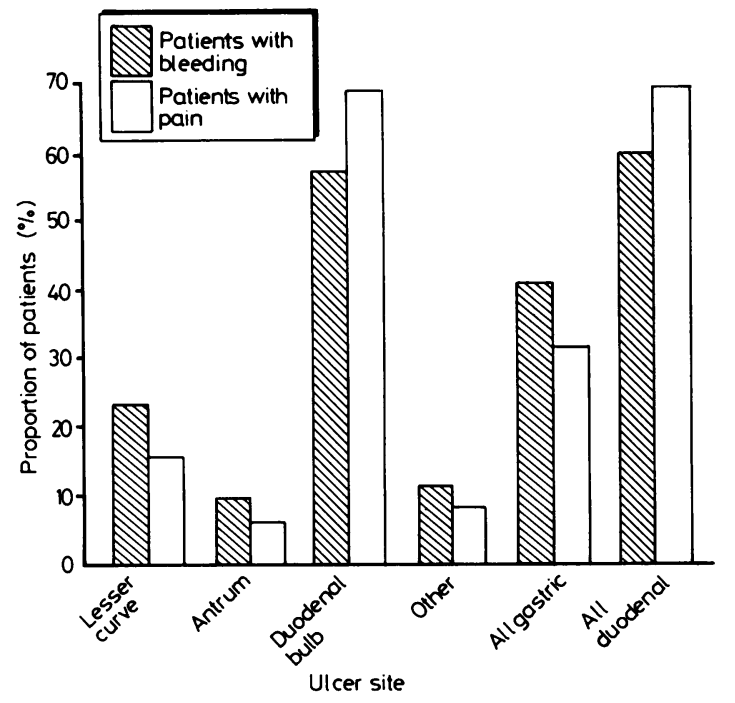

Fig. 4 Distribution of ulcer sites in patients with bleeding and pain.

were considered together $\left(13 \% \vee v 4 \%, \chi^{2}=7 \cdot 7\right.$, $\mathrm{p}<0 \cdot 01)$, but there was no such association with any other ulcer site.

Stigmata of recent haemorrhage was observed more frequently in patients presenting with bleeding rather than with pain $\left(79 \% \quad v 21 \%, \chi^{2}=63.4\right.$, $\mathrm{p}<0 \cdot 001)$. This difference was entirely accounted for by the presence of visible vessels $(52 \% \vee 0 \%$, $\left.\chi^{2}=47 \cdot 3, p<0 \cdot 001\right)$ and overlying clot $(10 \% v 1 \%$, $\chi^{2}=1 \cdot 6$, NS). In the absence of important SRH (visible vessel or clot) there was no difference in the frequency of red or black spots in the ulcer base $(17 \%$ $v 20 \%, \chi^{2}=0 \cdot 6$, NS). Considering all principal ulcers, the median diameter of ulcers which contained important SRH was significantly greater than those which did not $(11 \mathrm{~mm} v 9 \mathrm{~mm}, \mathrm{p}<0 \cdot 01)$ and there was a strong non-significant trend when bleeding ulcers were considered alone $(11.5 \mathrm{~mm} v 10 \mathrm{~mm}$, NS).

\section{Discussion}

The most striking difference between our two groups of patients was the difference in NSAID use during the four weeks before the endoscopy. This difference was apparent both for NANSAID and casual aspirin consumption. There is already strong evidence supporting an association between NANSAID consumption and bleeding peptic ulcer when compared with subjects not known to have peptic ulcer, ${ }^{134}$ and there is evidence for an association between regular heavy aspirin and uncomplicated gastric but not duodenal ulceration. ${ }^{5-7}$ Our prospective data suggest that there is a specific association between NSAID use and ulcers presenting with bleeding rather than with pain. It has been suggested that the association between aspirin use and gastrointestinal bleeding has been overestimated, and that some of the patients actually take aspirin for the symptoms of the peptic ulcer or haemorrhage. ${ }^{x}$ The study upon which this suggestion was based used community controls who were not known to have peptic ulceration. In only two of our patients presenting with bleeding who had used aspirin was this limited to the day of admission or the day before admission. Furthermore, our control group (those presenting with pain only), had at least as good a reason to use analgesics, and the association that we identified is therefore likely to be real.

Patients with bleeding were significantly older than patients with pain. Whilst this is probably a true difference, there are a number of potentially confounding factors. There might have been a tendency to avoid endoscoping elderly patients who presented to the hospital outpatient departments with pain alone, whereas there was no such reticence when patients presented with an acute haemorrhage. This seems unlikely as there is a fairly aggressive endoscopy policy in both hospitals. A more likely source of error is that local general practitioners were less inclined to refer elderly patients with pain than elderly patients with bleeding. Conversely, there may have been a greater readiness to refer patients with pain who were using NSAIDs. The patients presenting with bleeding who admitted to pain on specific questioning had presumably not had it sufficiently severely or for sufficiently long to seek medical advice. Absence of pain might be expected to lead to presentation with a complication and the observed tendency for the elderly to be pain free could be linked with the finding that the patients presenting with bleeding tended to be older.

Whilst there was no significant difference in the frequency of a previous ulcer diagnosis in the two groups, the data on the mode of presentation of those who had a previous ulcer diagnosis suggest that patients with complicated ulcers who develop recurrent ulceration tend to represent with a complication rather than with pain. The data also suggest that the previously recognised association of peptic ulceration with smoking ${ }^{2}$ is specific for those presenting with pain rather than with bleeding.

The greater frequency of large ulcers amongst those with bleeding is perhaps not surprising, as presumably the larger the ulcerated area the greater the risk of eroding through an artery, and this is supported by our data on visible vessels, overlying clot and ulcer size. Nor was it surprising that the frequency of visible vessels and overlying clot was significantly greater in patients presenting with 
bleeding rather than with pain, although it should be noted that there is a degree of subjectivity in the recognition of the visible vessel and there may be a greater inclination to diagnose them in patients known to have been admitted with acute bleeding. It was of interest that similar proportions of patients with bleeding and with pain had red or black spots in the base of their ulcers. This is consistent with our observation in a large prospective study" that these lesions, in contrast with visible vessels and clot in the base of the ulcer, are not associated with an increased risk of rebleeding after hospital admission. It seems likely that these simply occur randomly in ulcers regardless of presentation, and should no longer be regarded as stigmata of recent acute haemorrhage.

We conclude that there were identifiable differences in these two groups of patients whose peptic ulcers presented primarily with bleeding or pain. Patients presenting with bleeding were older; they were three times as likely to have taken an NSAID during the four weeks before diagnosis; they were more likely to have had a previous ulcer complication and more likely to have a large ulcer. The presence of one or more of these features in a patient who develops a peptic ulcer appears to be associated with a greater likelihood of presentation with a gastrointestinal haemorrhage.

Dr Matthewson was supported by a grant from the
Office of the Chief Scientist of the Department of Health and Social Security.

\section{References}

1 Somerville K, Faulkner G, Langman M. Non-steroidal anti-inflammatory drugs and bleeding peptic ulcer. Lancet 1986; i: 462-4.

2 Ainley CC, Forgacs IC, Keeling PWN, Thompson RPH. Outpatient endoscopic survey of smoking and peptic ulcer. Gut 1986; 27: 648-51.

3 Caradoc-Davies TH. Non-steroidal anti-inflammatory drugs, arthritis, and gastrointestinal bleeding in elderly in-patients. Age Ageing 1984; 13: 295-8.

4 O'Brien JD, Burnham WR. Bleeding from peptic ulcers and use of non-steroidal anti-inflammatory drugs in the Romford Area. Br Med J 1985; 291: 1609-10.

5 Gillies MA, Skyring A. Gastric and duodenal ulcer: the association between aspirin ingestion, smoking and family history of ulcer. Med J Austr 1969; ii: 280-4.

6 Duggan JM. Aspirin in chronic gastric ulcer: an Australian experience. Gut 1976; 17: 378-84.

7 Piper DW, McIntosh JH, Ariotti DE, Fenton BH, MacLennan R. Analgesic ingestion and chronic peptic ulcer. Gastroenterology 1981; 80: 427-32.

8 Coggon D, Langman MJS, Spiegelhalter D. Aspirin, paracetamol and haematemesis and melaena. Gut 1982; 23: $340-4$.

9 Swain CP, Storey DW, Bown SG, et al. Nature of the bleeding vessel in recurrently bleeding gastric ulcers. Gastroenterology 1986; 90: 595-608. 